\title{
Optimization of Rayleigh-Limited WDM-PONs With Reflective ONU by MUX Positioning and Optimal ONU Gain
}

\author{
Eduardo Tommy López, J. A. Lázaro, Member, IEEE, C. Arellano, V. Polo, and J. Prat, Member, IEEE
}

\begin{abstract}
In this work, the influence of the distribution element position and the reflective optical network unit (ONU) gain in wavelength-division-multiplexing passive optical networks are reported. After the theoretical and experimental analysis, it is determined that the best crosstalk-to-signal ratio is achieved if the multiplexer is placed either in the ONU or optical light termination vicinity, and the ONU gain has a new optimum value depending on the position. Also, it is demonstrated that Rayleigh backscattering is generally irrelevant in downstream transmission.
\end{abstract}

Index Terms-Access networks, optical fiber communication, passive optical networks (PONs), Rayleigh scattering, wavelength-division multiplexing (WDM).

\section{INTRODUCTION}

$\mathbf{R}$ ECENT developments in passive optical networks (PONs) propose to employ a reflective structure at the optical network unit (ONU), with centralized seeding light at the optical light termination (OLT) [1]. The carrier signal $(\mathrm{CW})$ is modulated with the user data at the ONU and back reflected in the upstream direction on the same wavelength. In this full-duplex single-fiber bidirectional transmission context, Rayleigh backscattering (RB) is the dominant impairment [2]. The aim of this work is to analyze the penalties in the upstream and downstream paths due to RB signals, extending our preliminary study [3], establishing the most adequate location of the multiplexer (MUX) in wavelength-division-multiplexing (WDM)-PONs, and the optimum ONU gain. Other effects, like the RB gain saturation [4] and the variation of the bandwidth of the receiver and the optical filter [5], are not considered here. An extension to time-division-multiplexing (TDM)-PON is under investigation and first results can be found in [6].

\section{THEORETICAL BASIS}

\section{A. RB Analysis in Upstream Path}

In WDM-PON, the MUX in the fiber path separates the wavelength channels, introducing a fixed attenuation. This splits the RB analysis into two zones: the feeder section (RB OLT-MUX)

Manuscript received September 18, 2009; revised October 16, 2009; accepted October 17, 2009. First published November 06, 2009; current version published January 07, 2010. This work was supported by the Spanish MICINN project TEC 2008-01887 TEYDE, the Alban Program, under scholarship E05D056349BR, by the Ramon y Cajal Program, by the European FP7 EURO-FOS Project, and by the SARDANA Project.

E. T. López, J. A. Lázaro, V. Polo, and J. Prat, are with the Optical Communications Group (GCO), Signal Theory and Communications Department (TSC), Universitat Politècnica de Catalunya (UPC), Barcelona 08034, Spain (e-mail: jprat@tsc.upc.edu).

C. Arellano is with VPIphotonics GmbH, D-10587 Berlin, Germany.

Color versions of one or more of the figures in this letter are available online at http://ieeexplore.ieee.org.

Digital Object Identifier 10.1109/LPT.2009.2035326

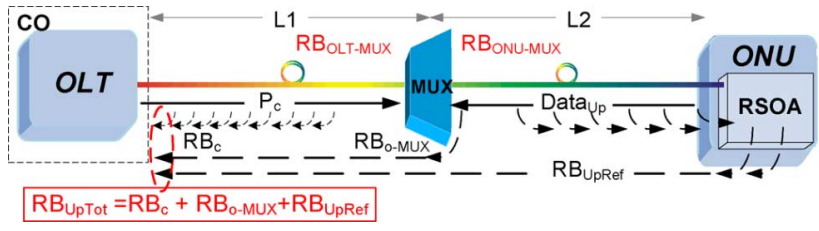

Fig. 1. Schematic of a plain WDM-PON with MUX at the fiber plant.

and the drop section ( $\mathrm{RB}_{\text {ONU-MUX }}$ ), as shown in Fig. 1. The crosstalk $(C)$, defined as the RB power at the OLT input, is the incoherent sum of the several RB contributions [2]: the RB generated by the downstream carrier signal at the OLT-MUX section $\left(\mathrm{RB}_{c}=P_{c} \cdot B\left(1-l_{1}^{2}\right)\right)$, the Rayleigh produced at the MUX output $\left(\mathrm{RB}_{o-\mathrm{MUX}}=P_{c} \cdot B\left(1-l_{2}^{2}\right) \cdot A^{2} \cdot l_{1}^{2}\right)$, and the RB generated by the upstream signal, reflected and reamplified by the ONU and transmitted to the OLT ( $\left.\mathrm{RB}_{\mathrm{UpRef}}\right)$, which dominates in the drop side, although it is reduced in one order of magnitude lower (twice the insertion losses of the MUX), with $\mathrm{RB}_{\mathrm{UpRefl}}=P_{o-\mathrm{ONU}} \cdot B\left(1-l_{2}^{2}\right) \cdot\left(g \cdot l_{1} \cdot l_{2} \cdot A\right)$, where $l_{1}$ and $l_{2}$ are the fiber losses in the feeder and the drop sections, respectively $\left(l_{n}=e^{-\alpha \cdot L n}\right), L_{n}$ is the corresponding fiber length ( $L=50 \mathrm{~km}), g$ is the ONUgain, $P_{o-\mathrm{ONU}}$ is the ONU output power $\left(P_{o \text {-ONU }}=P_{c} \cdot\left(g \cdot l_{1} \cdot l_{2} \cdot A\right)\right), P_{c}$ as the OLT output power, $A$ is the MUX insertion loss, $\alpha$ is the fiber attenuation, and $B$ is the Rayleigh coefficient (here $3.2 \times 10^{-5} \mathrm{~km}^{-1}$ ) [2]. The resulting crosstalk-to-signal $(\mathrm{C} / \mathrm{S})$ ratio or $\mathrm{OSRR}^{-1}$ (inverse optical signal-to-Rayleigh ratio), is then

$$
\left[\frac{\mathrm{C}}{\mathrm{S}}\right]=B \frac{\left(1-l_{1}^{2}\right)+\left(1-l_{2}^{2}\right) \cdot A^{2} \cdot l_{1}^{2}+\left(l_{1} l_{2} A\right)^{2}\left(1-l_{2}^{2}\right) g^{2}}{\left(l_{1} l_{2} A\right)^{2} g} \text {. }
$$

The second term is generally negligible, as attenuated by $A^{2}$. Fig. 2(a) shows theoretical C/S as a function of the feeder length for ONU gain of 10,15 , and $20 \mathrm{~dB}$. It is observed that there is a strong dependency on the gain, especially at the edges. Thus, it is found relevant to minimize the $\mathrm{C} / \mathrm{S}$ ratio; an optimal gain $g_{\text {opt }}$ as a function of the MUX position can be obtained by $\partial(\mathrm{C} / \mathrm{S}) / \partial g=0$, resulting in

$$
g_{\text {opt }}=\frac{1}{l_{\text {total }}} \sqrt{\frac{\left(1-l_{1}^{2}\right)}{\left(1-l_{2}^{2}\right)}} .
$$

With the MUX in an intermediate position, the optimum gain is equal to the total link loss $l_{\text {total }}=l_{1} l_{2} A$ (here, $15 \mathrm{~dB}$ ). If the MUX is in the ONU vicinity, the optimum ONU gain values increases to about $20 \mathrm{~dB}$ and higher. In contrast, if the MUX is near the OLT, a low ONU gain between 10 and $15 \mathrm{~dB}$ improves the C/S performance. Fig. 2(b) plots the optimal gain versus MUX position, for different MUX loss $(A)$ values. We observe a wide range around the intermediate position where 


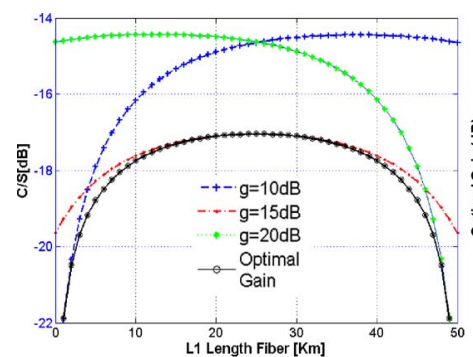

(a)

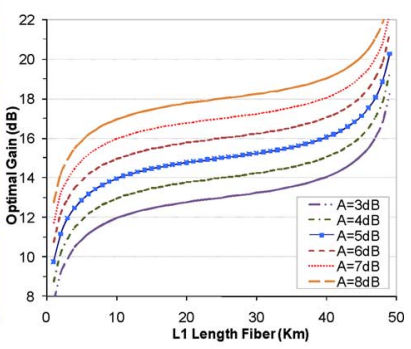

(b)
Fig. 2. (a) Theoretical $\mathrm{C} / \mathrm{S}$ as a function of the feeder length for ONU gain of 10,15 , and $20 \mathrm{~dB}$ and for the optimal gain [(2) in 1], with $\alpha(\mathrm{dB})=0.2 \mathrm{~dB} / \mathrm{km}$, $L 1+L 2=50 \mathrm{~km}, A=5 \mathrm{~dB}$, optical distribution network (ODN) total loss = $15 \mathrm{~dB}$. (b) Optimal gain as function of the distance to different values of $A$.

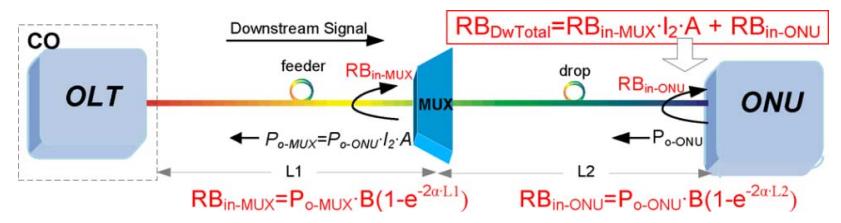

Fig. 3. Scenario for the downstream analysis.

the optimum value is about the link loss. At the edges, the performances improved, although the behavior gets rather critical with the gain selection. The worst positions for the MUX as a function of the gain are obtained from (1)

$$
\frac{\partial\left(\frac{C}{S}\right)}{\partial l_{1}}=0 \Rightarrow L_{1}=\frac{1}{4 \alpha} \cdot \ln \left(\frac{1}{A^{2} \cdot g^{2}}\right)+L .
$$

For $g=10,15$, and $20 \mathrm{~dB}$, maximal C/S are found in $L_{1}=$ $37.5,25$, and $12.5 \mathrm{~km}$, respectively.

In Fig. 2(a), the C/S with optimum gain is also plotted; $\mathrm{C} / \mathrm{S}$ minima correspond to MUX close to the OLT or close to the ONU. It is preferred that the MUX close to the ONU as for this case, the optimal gain is higher than the link losses, providing a valuable power margin.

\section{B. RB Analysis for Downstream}

For this analysis, the OLT generates a wavelength carrier intensity modulated with a nonreturn-to-zero (NRZ) data signal.

From Fig. 3, the RB at the ONU input is given by

$$
R B_{\text {DwTotal }}=P_{o-\mathrm{ONU}} \cdot B \cdot\left[\left[l_{2}{ }^{2} \cdot A^{2}\left(1-l_{1}^{2}\right)+\left(1-l_{2}^{2}\right)\right]\right. \text {. }
$$

From (4), it is observed that the $\mathrm{RB}_{\text {DwTotal }}$ depends more on the RB due to ONU output power, where a considerable reduction of the $\mathrm{RB}_{\text {in-MUX }}$ is received at the ONU, about one order of magnitude lower (twice the insertion losses of the MUX) than the $\mathrm{RB}_{\text {in-ONU }}$ at the drop section. So, when $l_{2}$ is maximum (MUX at the OLT), then $\mathrm{RB}_{\text {DwTotal }} \approx \mathrm{RB}_{\text {in-ONU. In this lo- }}$ cation, $\mathrm{RB}_{\text {in-ONU }}$ is maximal too and $\mathrm{RB}_{\text {DwTotal }}$ becomes the relevant expression, when the ONU gain is above the link loss. The optimum case is when the MUX is near ONU, $L_{2} \rightarrow 0$, and $\mathrm{RB}_{\text {DwTotal }}$ is minimal.

Simulations were performed using the VPI Transmission Maker, at a data rate of $1.25 \mathrm{~Gb} / \mathrm{s}$. The OLT consists of a CW laser, a Mach-Zehnder modulator, a circulator, and an avalanche photodiode (APD). OLT output power is fixed to $0 \mathrm{dBm}$. ODN is composed of $50 \mathrm{Km}$ of Universal Fiber with

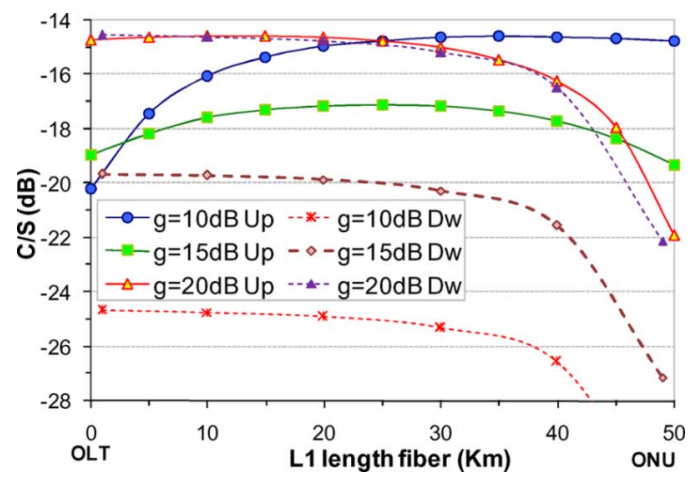

Fig. 4. C/S as a function of the MUX position for ONU gain of 10, 15, and $20 \mathrm{~dB}$ in downstream and upstream transmission.

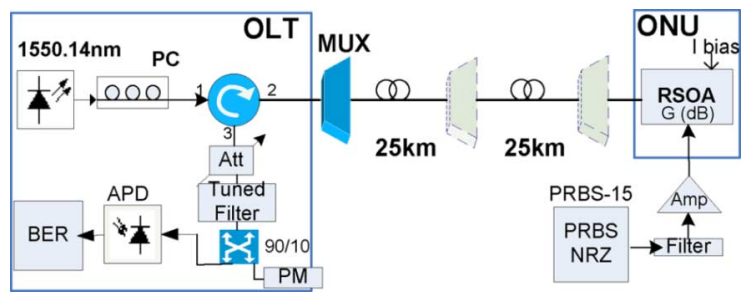

Fig. 5. Setup for the experimental measurements.

$0.2 \mathrm{~dB} / \mathrm{Km}$ of attenuation and a WDM MUX with $5 \mathrm{~dB}$ of losses. The ONU is formed by an electroabsorption modulator $(\mathrm{EAM})+$ semiconductor optical amplifier (SOA) like reflective-SOA (RSOA).

Simulations were performed on both, unmodulated carrier and down data transmission. Results in terms of the RB power level and bit-error rate (BER) are the same; although the spectral distributions are different, they overlap. The experimental test will consider the worst case: the distribution of a CW carrier, corresponding to a narrower RB spectra and then affecting more to the BER [7].

Fig. 4 shows obtained C/S for ONU gains of 10, 15, and $20 \mathrm{~dB}$ for up-down transmission. In upstream the $\mathrm{C} / \mathrm{S}$ ratio agrees with theoretical Fig. 2(a). In downstream, C/S ratios are better than the ones obtained in upstream, and depend on the ONU gain. This dependence does not affect the BER performance. The best position for the MUX is near ONU. These results demonstrate that the RB power in downstream is negligible, and only becomes relevant when the ONU gain is above the ODN losses with the MUX positioned furthest from the ONU, as also will be shown in Fig. 7.

\section{EXPERIMENTAL MEASURES AND RESUlTS}

Fig. 5 shows the experimental setup. At the OLT, a tunable laser was used to feed the ONU at $1550.14 \mathrm{~nm}$, matching the MUX channel (5-dB insertion loss). The power applied to the feeder fiber was $0 \mathrm{dBm}$. The upstream reception was carried out by an APD (sensitivity of $-22.5 \mathrm{dBm}$ for BER $=10^{-10}$ ). An optical filter (BW $34.5 \mathrm{GHz}$ ) was introduced before the photodetection. A variable attenuator was used to obtain the sensitivity curve (Fig. 6). The link was formed by two fiber sections of $25 \mathrm{~km}$.

The ONU was formed by a Kamelian RSOA 18-TO-37-08, directly modulated with the upstream data at $1.25 \mathrm{~Gb} / \mathrm{s}$ $\left(\mathrm{PRBS} 2{ }^{15}-1, \mathrm{ER}=11 \mathrm{~dB}\right)$. The RSOA gain values $(10,15$, 


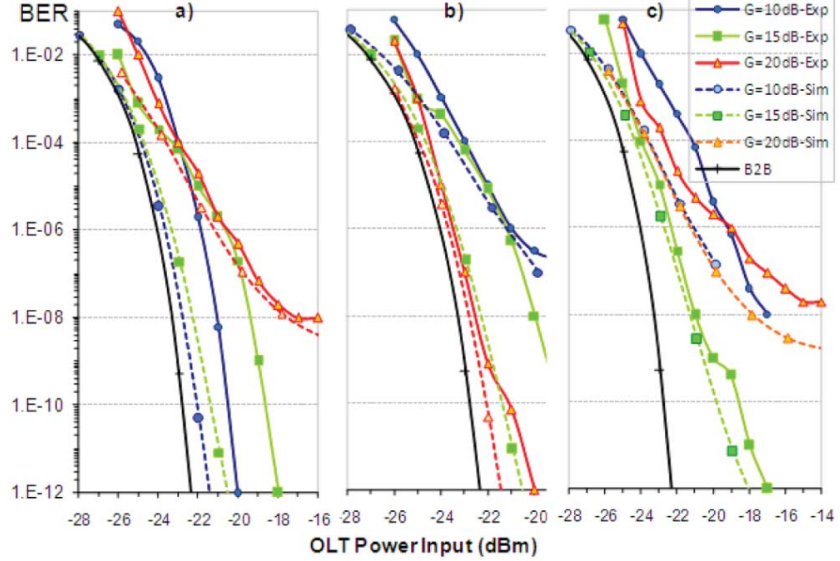

Fig. 6. BER measurements for 10,15 , and $20 \mathrm{~dB}$ of ONU gain with the MUX (a) at the OLT side, (b) at the ONU side, (c) at half way between OLT and ONU. Experimental and simulated curves.

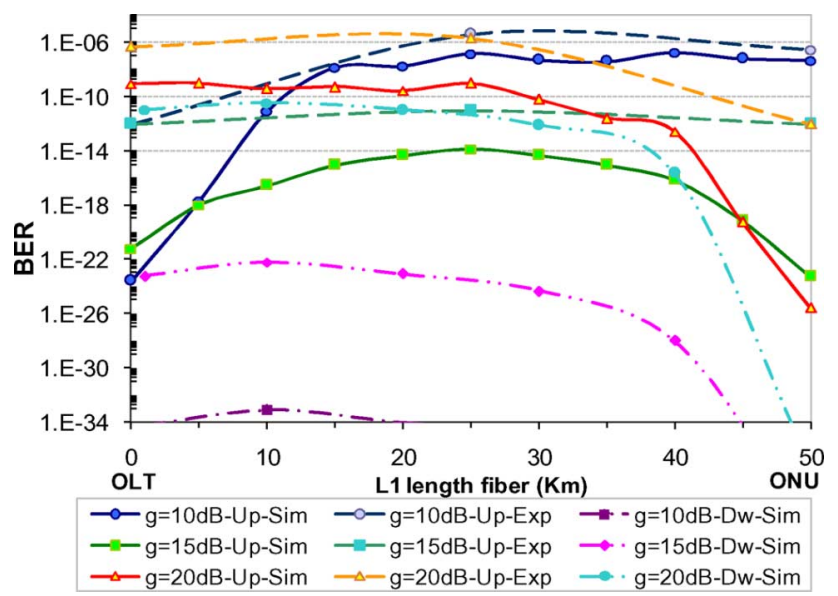

Fig. 7. BER as a function of the MUX position to different ONU gain values (simulations and experimental results).

and $20 \mathrm{~dB}$ ) were characterized as a function of the bias current $(34,45$, and $80 \mathrm{~mA})$.

Several measurements were carried out for different MUX positions: at the OLT side $(L 1=0 \mathrm{~km}, L 2=50 \mathrm{~km})$; at the ONU premises ( $L 1=50 \mathrm{~km}, L 2=0 \mathrm{~km})$; and at half-way of the link $(L 1=25 \mathrm{~km}, L 2=25 \mathrm{~km})$. Back-to-back measurement was performed too (Fig. 6). These measurements are compared to the simulation results, performed in the same power and conditions, based on GPON standard, with similar results.

With the MUX near the OLT, the ONU gain of $10 \mathrm{~dB}$ performs the best (2.2-dB penalty), while the worst case is for a gain of $20 \mathrm{~dB}$, with a $B E R=10^{-8}$ error floor. This result is due to the high influence of the $\mathrm{RB}_{\mathrm{UpRef}}$ at the ONU.

At the ONU side, higher amplification improves the system. The penalty for $G=20 \mathrm{~dB}$ is $1.5 \mathrm{~dB}$. In this case, there is an error floor of the order of $B E R=10^{-7}$ for the lowest gain $(10 \mathrm{~dB})$, since $\mathrm{RB}_{c}$ is the limiting and the upstream signal is weak. The reflected RB is not relevant due to the double attenuation by the MUX and the feeder fiber.

For the MUX located in the middle of the link, performances are, in general, worse, because the $25 \mathrm{~km}$ of fiber on each side are long enough for the RB generation in both sections. $\mathrm{RB}_{c}$ is the most relevant for low ONU gain while $\mathrm{RB}_{\mathrm{UpRef}}$ is for high gain. In this case, the ONU gain equal to the link loss is the best (penalty of $3.9 \mathrm{~dB}$ ).

The BER in upstream and downstream for different values of ONU gain is also shown in Fig. 7 (simulation/experimental). We observe that at any position of the MUX, with an ONU gain recovering the ODN loss (15 dB), the BER performance is always better than $10^{-10}$, at the RX sensitivity specified. However, for low or high gain values (e.g., 10 and $20 \mathrm{~dB}$, respectively), it is possible to be below the required BER only if selecting the MUX location properly. For an ONUgain $=20 \mathrm{~dB}$, it is better to place the MUX within $10 \mathrm{~km}$ from the ONU, since longer drop fiber will highly increase C/S. Gain values smaller than $10 \mathrm{~dB}$ are not recommendable because of the receiver noise. For the input powers received at the RSOA $(\approx-15 \mathrm{dBm})$, no significant gain saturation of the RSOA is observed that could potentially reduce the crosstalk [7], [4].

The upstream BER curves follow the same tendency as the simulation; some differences are due to the limited RX sensitivity, to the difficulty measuring BER values better than $10^{-12}$ during real tests, and also to the simulation model where only $\mathrm{RB}$ noise was taken into account.

\section{CONCLUSION}

We demonstrated that the Rayleigh crosstalk signals substantially vary depending on the position of the distribution element, since they are determined by the length of the fiber and by the ONU gain applied. Although, in a real-world deployment, the position of the distribution element is usually determined by more practical considerations, like the cost, the physical distribution of the customers, etc., this study provides relevant information in terms of transmission optimization in WDM-PON. The results revealed that the best performance can be achieved if the distribution element is placed either in the ONU or OLT vicinity, at the expense, in such cases, that the ONU gain takes a new optimum depending on that exact position. Also, it was demonstrated that the downstream RB power is generally irrelevant for the data transmission.

\section{REFERENCES}

[1] J. Prat, C. Arellano, V. Polo, and C. Bock, "Optical network unit based on a bidirectional reflective semiconductor optical amplifier for fiber-to-the-home networks," IEEE Photon. Technol. Lett., vol. 17, no. 1, pp. 250-252, Jan. 2005.

[2] C. Arellano, K.-D. Langer, and J. Prat, "Reflections and multiple Rayleigh backscattering in WDM single-fiber loopback access networks," J. Lightw. Technol., vol. 27, no. 1, pp. 12-18, Jan. 2009.

[3] C. Arellano, V. Polo, and J. Prat, "Effect of the multiplexer position in Rayleigh-limited WDM-PONs with amplified-reflective ONU," in 9th ICTON '07, Jul. 1-5, 2007, vol. 4, pp. 84-87.

[4] E. MacHale et al., "Signal-induced Rayleigh noise reduction using gain saturation in an integrated R-EAM-SOA," in OFC 2009 Conf., Mar. 22-26, 2009, pp. 1-3, Paper OThA6.

[5] G. Talli, C. Chow, and P. D. Townsend, "Modeling of modulation formats for interferometric noise mitigation," J. Lightw. Technol., vol. 26, no. 17, pp. 3190-3198, Sep. 1, 2008.

[6] E. Lopez, J. Lazaro, V. Polo, and J. Prat, "Optimal gain and position of the distribution element in Rayleigh-limited WDM and TDM PONs with reflective ONU," in ECOC 2009 Conf., Sep. 21-24, 2009, P6.27.

[7] J. A. Lazaro, C. Arellano, and J. Prat, "Rayleigh scattering reduction by means of optical frequency dithering in passive optical networks with remotely seeded ONUs," IEEE Photon. Technol. Lett., vol. 19, no. 2, pp. 64-66, Jan. 15, 2007. 